\title{
Comparison of three cell block techniques for detection of low frequency abnormal cells
}

\author{
Steven A Hecht \\ Matthew McCormack \\ Hologic Inc, Marlborough, MA, USA
}

Correspondence: Steven A Hecht Hologic Inc, 250 Campus Drive, Marlborough, MA 01752, USA

Tel +I 5082638694

Fax +I 5082632970

Email steven.hecht@hologic.com

\author{
This article was published in the following Dove Press journal: \\ Pathology and Laboratory Medicine International \\ 3 January 2013 \\ Number of times this article has been viewed
}

\begin{abstract}
Background: The Cellient ${ }^{\circledR}$ Automated Cell Block System rapidly creates paraffin-embedded cell blocks by using vacuum filtration to deposit a layer of cells on a filter and infiltrate those cells with reagents and paraffin. This study used a "tracer" cell model to mimic low frequency abnormal cells and compare detection and representative sampling with simple sedimentation, Richard-Allan HistoGelTM, and Cellient cell block techniques.
\end{abstract}

Methods: Tracer cells were a cultured cell line (CaSki) fixed in methanol, prestained in solution with hematoxylin, and quantified using a hemacytometer. Tracer cells were diluted in a 10-fold dilution series ranging from 100 to 0.1 tracer/mL in a background of pooled clinical serous effusion specimens. Ten replicates of each dilution were processed using each cell block method, and the resulting blocks were cut to produce two slides from each block. The slides were deparaffinized, counterstained with eosin, cover-slipped, and screened for the presence of tracer cells. Blocks were considered to be representative of the specimen if tracer cells were detected on either of the slides. If no tracer cells were observed on either slide, the block was recut to generate a third slide. If tracer cells were seen on the third slide, the block was considered representative of the specimen.

Results: Tracer cells were identified on the initial slides for 20 of $40(50.0 \%)$ simple sedimentation, 21 of 40 (52.5\%) of HistoGel, and 25 of 40 (62.5\%) of Cellient cell blocks. Representative sampling of the $1 \mathrm{tracer} / \mathrm{mL}$ specimen was $10.0 \%$ for simple sedimentation and $30.0 \%$ for HistoGel and Cellient. Only Cellient showed representative sampling of the 0.1 tracer $/ \mathrm{mL}$ specimen.

Conclusion: The Cellient System blocks demonstrated representative sampling at the lowest tracer cell concentration compared with simple sedimentation and HistoGel.

Keywords: Cellient ${ }^{\circledR}$, HistoGel ${ }^{\mathrm{TM}}$, simple sedimentation, CaSki, microtomy

\section{Introduction}

Cell block techniques can be of value to anatomic pathologists when analyzing cytology specimens by enabling microscopic evaluation that can mimic histology. ${ }^{1,2}$ Cell blocks can also be useful in the evaluation of cytoarchitecture and microbiopsies, as well as for performing special stains and immunohistochemistry. ${ }^{3-5}$

Traditional cell block techniques, such as plasma-thrombin, collodion bag, and simple sedimentation, can be technically challenging to prepare and to section on a microtome. These techniques rely on formation of a clearly visible cell pellet after centrifugation, which limits their application for specimens of low cellularity. In addition, cell loss during long tissue processing cycles and uncertainty about the location of cells within the block can add complexity to cell block preparation using traditional methods. submit your manuscript $\mid$ www.dovepress.com

Dovepress

http://dx.doi.org// 0.21 47/PLMI.S37555
Pathology and Laboratory Medicine International 2013:5 I-7

(C) 2013 Hecht and McCormack, publisher and licensee Dove Medical Press Ltd. This is an Open Access article which permits unrestricted noncommercial use, provided the original work is properly cited. 
In contrast, the Cellient ${ }^{\circledR}$ Automated Cell Block System (Hologic Inc, Bedford, MA) creates a paraffin-embedded cell block by using vacuum filtration to deposit a layer of cells on a filter and infiltrate those cells with processing reagents and paraffin. ${ }^{6}$ Thus, Cellient has the potential to improve cell visualization during microtomy and microscopic evaluation because the cells are deposited in a defined region at the face of the block.

This study used a novel "tracer cell" model consisting of CaSki cells (American Type Culture Collection, Manassas, VA) prestained in solution with hematoxylin and serially diluted in a background of pooled clinical serous effusion specimens. The tracer cell model was used to evaluate the detection and representative sampling of low frequency abnormal cells using three cell block preparation methods, ie, simple sedimentation, Richard-Allan HistoGel ${ }^{\text {TM }}$ (HistoGel) (Thermo Scientific, Waltham, MA), and Cellient.

\section{Materials and methods}

A flow chart of the experimental methods is shown in Figure 1.

\section{Tracer cell production}

The CaSki cell line was cultured according to standard microbiological practice. The cells were harvested, transferred to $50 \%$ methanol for approximately 24 hours, and stained with hematoxylin in solution. Briefly, the cells were centrifuged from $50 \%$ methanol, washed once with phosphate-buffered saline, resuspended in Richard-Allan Hematoxylin 1 (Thermo Scientific) for 20-30 minutes on a rotary shaker, washed in 3-4 changes of phosphate-buffered saline, and then transferred to PreservCyt ${ }^{\circledR}$ solution (Hologic Inc). This concentrated solution of tracer cells was quantified using a hemacytometer counting chamber.

\section{Preparation of serous effusion specimens}

Eight serous effusion specimens (pleural, peritoneal, and ascites fluid) were processed into PreservCyt solution according to the ThinPrep ${ }^{\circledR} 2000$ Operator's Manual non-gynecological protocol and the Cellient Operator's Manual. ${ }^{7,8}$ Fifty milliliter aliquots of fresh effusion were centrifuged at $1200 \times \mathrm{g}$ for 10 minutes, and the pellets were washed once with $30 \mathrm{~mL}$ of CytoLyt $^{\circledR}$ (Hologic Inc) and centrifuged in the same manner. Up to 10 drops of the resulting pelleted specimen were added to a $20 \mathrm{~mL}$ vial of PreservCyt solution. The resulting vials were combined to obtain the volume of background cells required for use as diluent for the study.

\section{Dilution of tracer cells}

The tracer cell stock was seeded into a sufficient volume of the pooled serous effusion specimens to create a nominal concentration of 100 tracer cells per mL. Tracer cells were serially diluted 1:10 in a background of pooled serous effusion specimens in PreservCyt solution ranging from 100 to 0.1 tracer $/ \mathrm{mL}$. A sufficient volume of each dilution was made to allow filling of 30 vials with $20 \mathrm{~mL}$ of specimen. The 30 vials of each dilution were divided into three groups of 10 vials for processing by the three cell block methods, ie, simple sedimentation, HistoGel, and Cellient.

\section{Cell block preparation}

Ten $20 \mathrm{~mL}$ replicates of each tracer cell concentration were used to make the cell blocks by each method.

\section{Simple sedimentation}

Following the HistoGel package insert, the entire volume of each specimen was transferred to a $50 \mathrm{~mL}$ disposable centrifuge tube and centrifuged for five minutes at $2082 \times \mathrm{g}$. The supernatant was decanted by carefully inverting the tube, and the pellet was resuspended in $1 \mathrm{~mL}$ of eosin zinc formalin. The tube was centrifuged in the same manner, the supernatant was removed by aspiration, and the pellet was removed using a metal spatula then placed in the center of a $2 \times 2$ inch square of lens tissue. The lens tissue was gently folded over the cells, placed in a tissue processor cassette, and stored in zinc formalin until the tissue processor run.

\section{HistoGel}

The entire volume of each specimen was transferred to a $50 \mathrm{~mL}$ disposable centrifuge tube and centrifuged for five minutes at $2082 \times \mathrm{g}$. The supernatant was decanted by carefully inverting the tube and $200 \mu \mathrm{L}$ of liquefied HistoGel was added to the pellet. The pellet was resuspended in the HistoGel, and the tube was then placed on ice to solidify. The solidified HistoGel was placed in a tissue processor cassette and kept in zinc formalin until the tissue processor run.

\section{Tissue processor protocol}

An ASP 300 tissue processor (Leica Microsystems, International, Nussloch, Germany) was used to process the simple sedimentation and HistoGel specimens. After the tissue processor run was complete, specimens were embedded in fresh paraffin in routine fashion. The protocol for the processor is shown in Table 1.

\section{Cellient}

Cellient cell blocks were made according to the standard operating instructions for the instrument (Figure 2). ${ }^{6}$ Each $20 \mathrm{~mL}$ specimen was homogenized, decanted into a 

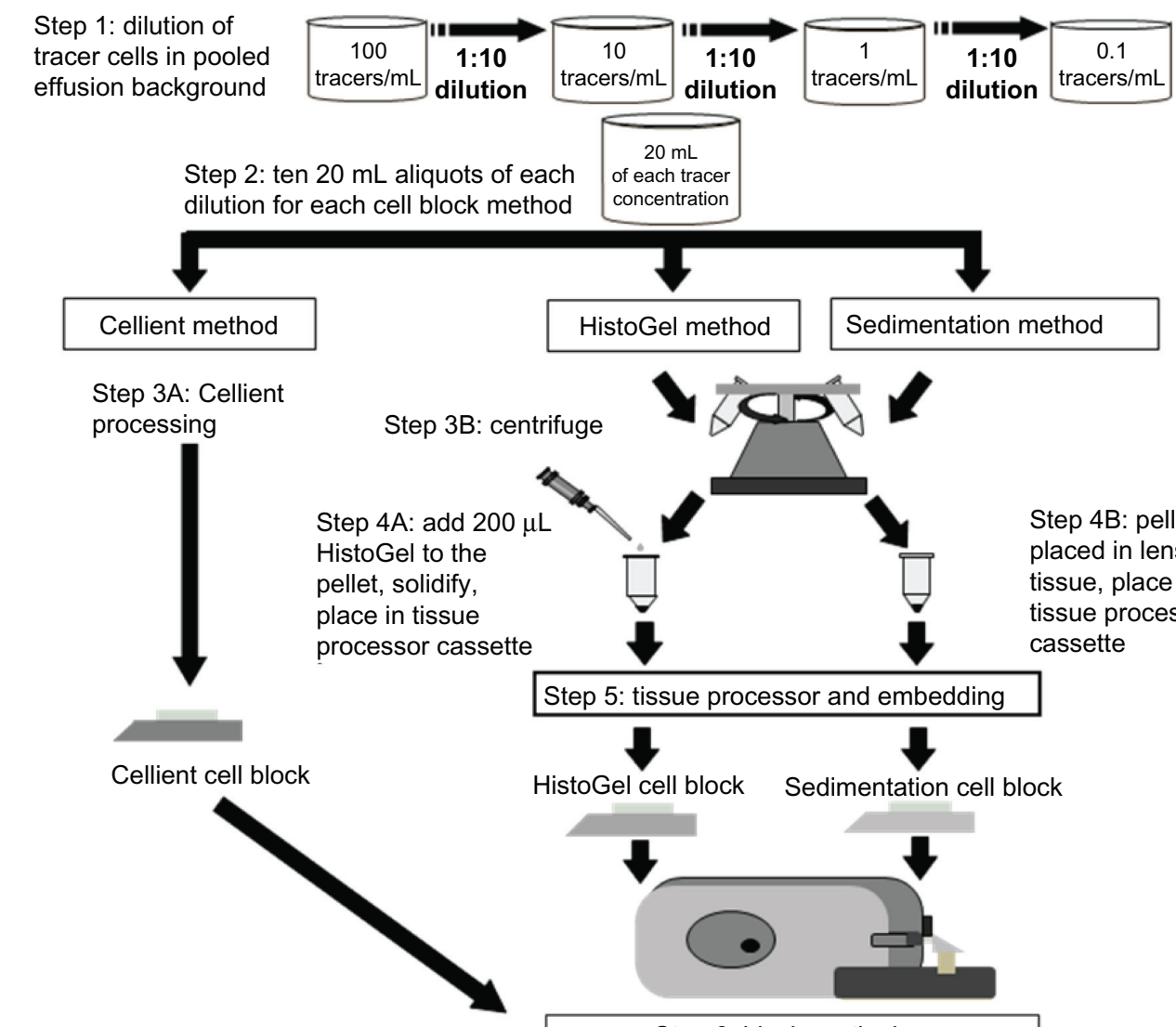

Step 3B: centrifuge

Step 4A: add $200 \mu \mathrm{L}$ HistoGel to the pellet, solidify, place in tissue processor cassette
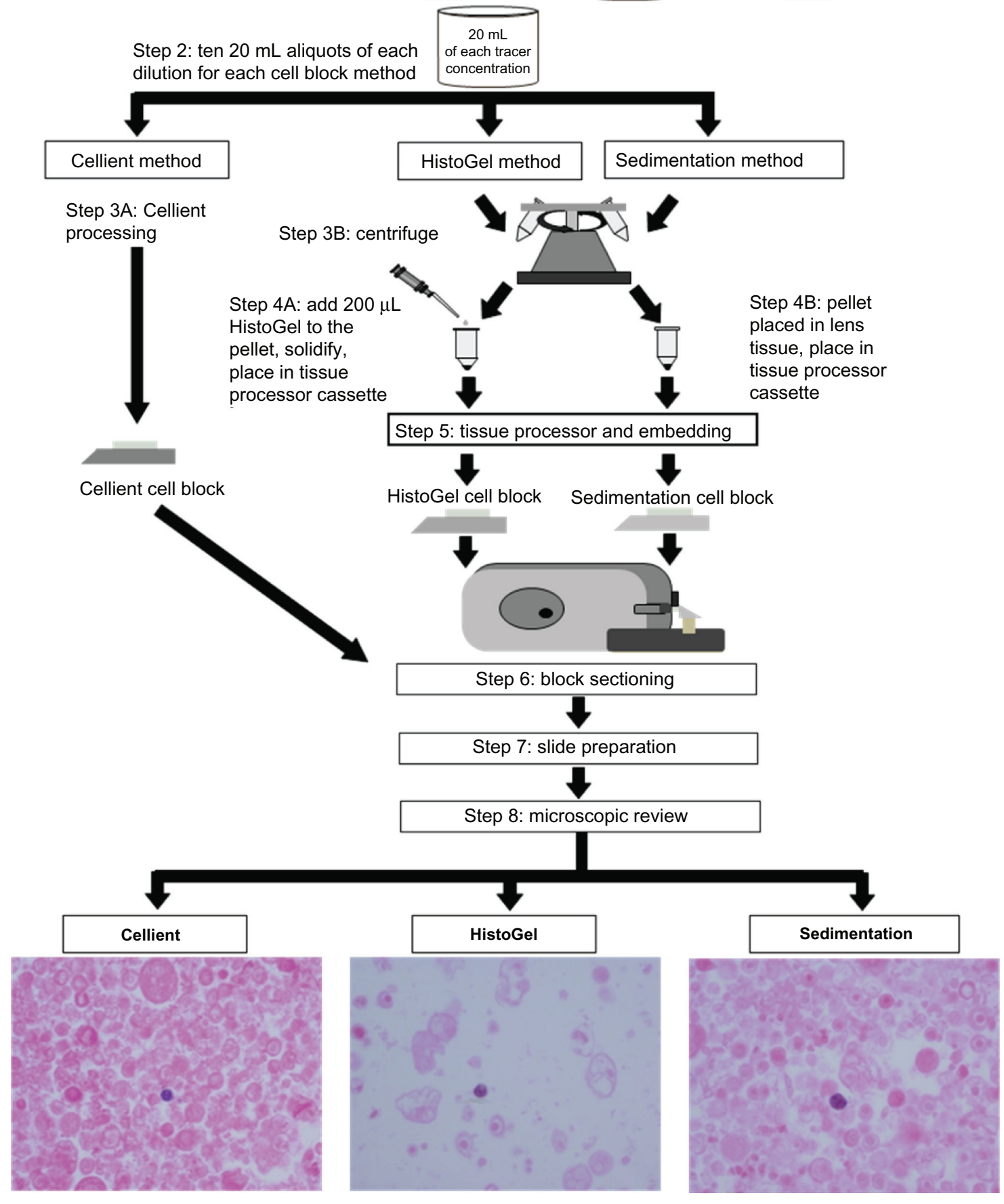

Figure I Flow chart of experimental methods.

Cellient-compatible vial, and loaded into the instrument. The system transferred the entire vial contents on a membrane filter and added approximately $200 \mu \mathrm{L}$ of eosin stain to aid in visualization of the cell spot. The instrument dehydrated the specimen with isopropyl alcohol, cleared it in xylene, and infiltrated it with molten paraffin wax. The instrument then solidified the paraffin by chilling the block. After completing the block using the Cellient finishing station, the cell block was ready for sectioning on the microtome. Three Cellient instruments were used.

\section{Microtomy and slide preparation}

All cell blocks were cut on a motorized rotary microtome (RM2255, Leica Microsystems) at $5 \mu \mathrm{m}$, generating two 
Table I Leica ASP 300 tissue processor protocol

\begin{tabular}{lllll}
\hline Step & Reagent & $\begin{array}{l}\text { Duration } \\
\text { (minutes) }\end{array}$ & $\begin{array}{l}\text { Temperature } \\
\left({ }^{\circ} \mathbf{C}\right)\end{array}$ & $\begin{array}{l}\text { Pressure } \\
\text { on/off }\end{array}$ \\
\hline 1 & Zinc formalin & 30 & Ambient & Off \\
2 & Zinc formalin & 55 & Ambient & Off \\
3 & $50 \%$ alcohol & 30 & 35 & Off \\
4 & $70 \%$ alcohol & 30 & 35 & Off \\
5 & $90 \%$ alcohol & 30 & 35 & Off \\
6 & $95 \%$ alcohol & 55 & 35 & Off \\
7 & I00\% alcohol & 50 & 35 & Off \\
8 & I00\% alcohol & 50 & 35 & Off \\
9 & Xylene & 55 & 40 & On \\
10 & Xylene & 55 & 40 & On \\
11 & Paraffin & 30 & 60 & On \\
12 & Paraffin & 30 & 60 & On \\
13 & Paraffin & 55 & 60 & On \\
\hline
\end{tabular}

slides per block and representing two levels of the block. The levels were approximately $50-100 \mu \mathrm{m}$ apart, except for Cellient, for which the two levels were 25-50 $\mu \mathrm{m}$ apart due to the thin nature of the Cellient cell blocks. Slides were deparaffinized and only counterstained with eosin to allow visualization of the blue tracer cells. The slides were processed on a Leica ST5020 autostainer (Leica Microsystems) using the program shown in Table 2. Coverslips were applied using a Leica CV5030 automated cover-slipper (Leica Microsystems) integrated with the autostainer.

\section{Tracer cell detection}

The slides were screened using a light microscope for the presence of tracer cells by a cytotechnologist who was blinded to the cell block method and tracer cell concentration. If tracer cells were seen on either of the two slides, the block was deemed representative of the specimen. If no tracer cells were seen on either slide, the block was recut to generate a third slide. If tracer cells were seen on this third slide, the block was then deemed representative of the specimen. If no tracer cells were seen on any of the three slides, the block was deemed not representative.

\section{Results}

Tracer cells were identified on the initial slides for 20 of $40(50.0 \%)$ simple sedimentation cell blocks, 21 of $40(52.5 \%)$ HistoGel cell blocks, and 25 of 40 (62.5\%) Cellient cell blocks. The numbers of cell blocks requiring recutting due to lack of tracer cells being identified on either of the two

A

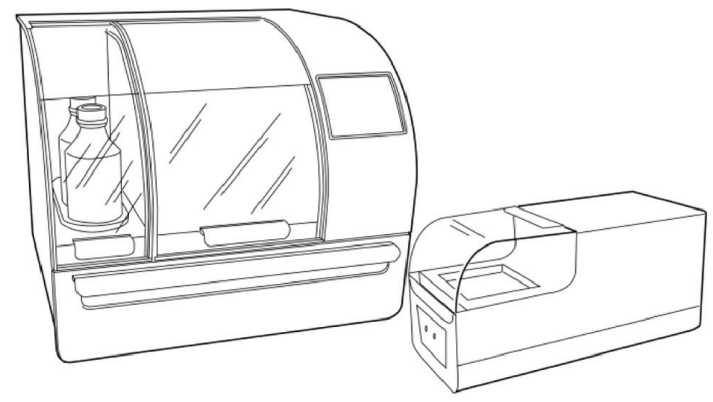

B

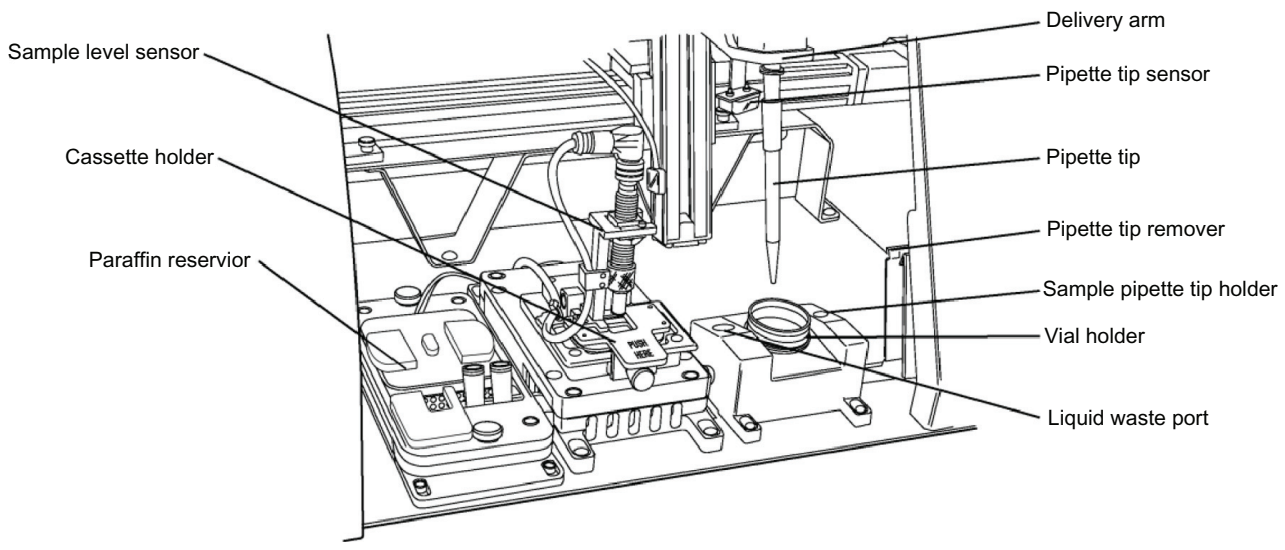

Figure 2 Cellient ${ }^{\circledR}$ Automated Cell Block System. (A) Automated Cell Block Processor and Finishing Station. (B) Processor compartment components. 
Table 2 Leica ST5020 autostainer modified staining protocol

\begin{tabular}{lll}
\hline Step & Reagent & Time \\
\hline 1 & Oven station at $70^{\circ} \mathrm{C}$ & 20 minutes \\
2 & Xylene & 5 minutes \\
3 & Xylene & 5 minutes \\
4 & $100 \%$ alcohol & 90 seconds \\
5 & $100 \%$ alcohol & 90 seconds \\
6 & $95 \%$ alcohol & 60 seconds \\
7 & Eosin & 10 seconds \\
8 & $95 \%$ alcohol & 60 seconds \\
9 & $100 \%$ alcohol & 62 seconds \\
10 & $100 \%$ alcohol & 60 seconds \\
11 & Xylene & 90 seconds \\
12 & Xylene & 90 seconds \\
\hline
\end{tabular}

initial slides are shown in Table 3. For simple sedimentation, recutting was required for one of 10 blocks at 10 tracer $/ \mathrm{mL}$, nine of 10 blocks at 1 tracer $/ \mathrm{mL}$, and 10 of 10 blocks at $0.1 \mathrm{tracer} / \mathrm{mL}$. Of the simple sedimentation recuts, one of the 20 blocks $(5.0 \%)$ from a 10 tracer $/ \mathrm{mL}$ specimen showed tracer cells (Table 4). The HistoGel method required recutting for two of 10 blocks at 10 tracer/mL, seven of 10 blocks at 1 tracer $/ \mathrm{mL}$, and 10 of 10 blocks at 0.1 tracer $/ \mathrm{mL}$. Two of the 19 HistoGel recuts $(10.5 \%)$ produced representative blocks at 10 tracer $/ \mathrm{mL}$ (Table 4). The Cellient method required recutting for seven of 10 blocks at 1 tracer/mL and eight of 10 blocks at 0.1 tracer $/ \mathrm{mL}$. One of 15 Cellient recut blocks (6.7\%) showed tracer cells at 1 tracer/mL (Table 4 ).

Representative sampling of all tracer cell dilutions for each cell block method is shown in Figure 3. All three methods showed representative sampling in $100 \%$ of the blocks made from the 100 tracer/mL specimen. With blocks made from the 10 tracer/mL specimen, simple sedimentation, HistoGel, and Cellient showed $90.0 \%, 80.0 \%$, and $100 \%$ representative sampling, respectively. Representative sampling at the 1 tracer/mL dilution was $10.0 \%$ for simple sedimentation and $30 \%$ for HistoGel and Cellient. At the 0.1 tracer $/ \mathrm{mL}$ dilution, only Cellient showed representative sampling

Table 3 Number of blocks requiring recutting

\begin{tabular}{llll}
\hline $\begin{array}{l}\text { Concentration } \\
\text { (tracer/mL) }\end{array}$ & $\begin{array}{l}\text { Simple } \\
\text { sedimentation } \\
(\mathbf{n = 4 0 )}\end{array}$ & $\begin{array}{l}\text { HistoGel } \\
(\mathbf{n}=\mathbf{4 0})\end{array}$ & $\begin{array}{l}\text { Cellient }^{\mathrm{B}} \\
(\mathbf{n}=\mathbf{4 0})\end{array}$ \\
\hline 0.1 & 10 & 10 & 8 \\
1 & 9 & 7 & 7 \\
10 & 1 & 2 & 0 \\
100 & 0 & 0 & 0 \\
Total & 20 & 19 & 15 \\
\hline
\end{tabular}

Note: Fewer Cellient cell blocks required recutting at tracer cell concentrations ranging from 0.1 to 10 tracer $/ \mathrm{mL}$ and in total compared with the simple sedimentation and HistoGel cell blocks.
Table 4 Number of recut blocks with tracer cells detected

\begin{tabular}{llll}
\hline $\begin{array}{l}\text { Concentration } \\
\text { (tracer/mL) }\end{array}$ & $\begin{array}{l}\text { Simple } \\
\text { sedimentation } \\
(\mathbf{n}=\mathbf{2 0})\end{array}$ & $\begin{array}{l}\text { HistoGel } \\
(\mathbf{n}=1 \mathbf{9})\end{array}$ & $\begin{array}{l}\text { Cellient }^{\mathrm{B}} \\
(\mathbf{n}=15)\end{array}$ \\
\hline 0.1 & 0 & 0 & 0 \\
1 & 0 & 0 & 1 \\
10 & 1 & 2 & 0 \\
100 & 0 & 0 & 0 \\
Total & $\mathrm{I}$ & 2 & $\mathrm{I}$ \\
\hline
\end{tabular}

Note: Tracer cells were detected at a lower concentration for Cellient cell blocks compared with the simple sedimentation and HistoGel cell blocks.

with $20.0 \%$ of the blocks. Representative images of simple sedimentation, HistoGel, and Cellient tracer cell blocks are shown in Figure 1 (magnification 400×).

\section{Discussion}

This study demonstrates that detection of low concentration CaSki tracer cells was greater in Cellient cell blocks compared with simple sedimentation and HistoGel cell blocks. The Cellient cell blocks required the least amount of recutting, and demonstrated representative sampling at the lowest tracer cell concentration.

Few studies have compared the performance of different cell block methodologies. ${ }^{3,9,10}$ A recent report by Gorman et al compared the results of immunohistochemical assays performed on thrombin, formalin, and Cellient cell blocks with those performed on resected tissue specimens from 31 cases of invasive breast cancer. ${ }^{9}$ Adequate cellularity was obtained in $31(100 \%)$ Cellient blocks, $25(80.6 \%)$ formalin blocks, and $23(74.2 \%)$ thrombin blocks. These results support the findings of the present study, in which greater numbers of low cellularity specimens were identified using the Cellient cell block than with other cell block methods.

Wagner et al compared the morphologic and immunohistochemical staining patterns of simple sedimentation cell blocks and Cellient cell blocks for 16 benign and 19 malignant non-gynecologic cytology specimens. ${ }^{10}$ For the benign cell blocks, adequate cellularity was achieved in all cases, and there were no significant morphologic differences between cell block methods. For the malignant cell blocks, a nonsignificant difference $(P<0.737)$ in cellularity was observed for Cellient compared with simple sedimentation cell blocks. These results contrast with the findings of the present study and those reported by Gorman et al. ${ }^{9}$

A benefit of the Cellient system is that it concentrates cellular material on a membrane filter, resulting in a cell block with all the cells at or near the face of the block. This increases the likelihood that cells will be captured during 


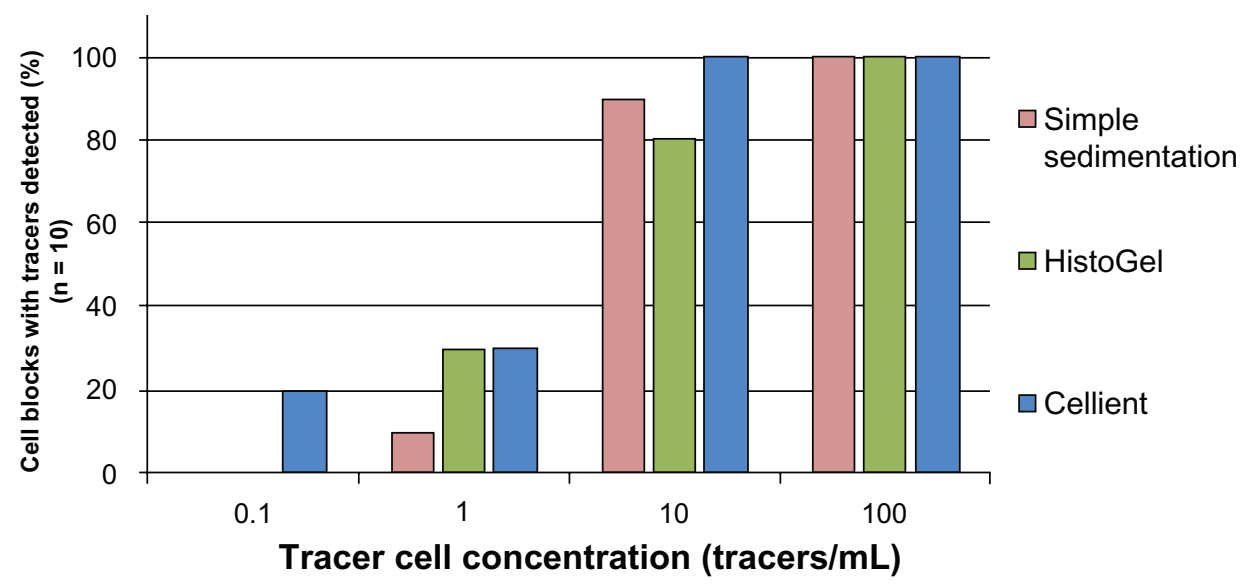

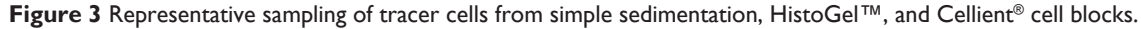

Note: The Cellient cell blocks demonstrated representative sampling at the lowest tracer cell concentration, 0.1 tracer/mL, which was not detected by the simple sedimentation and HistoGel cell blocks.

microtomy because all the cells are located in a defined region of the block. In contrast, cells may be randomly located throughout the block using the simple sedimentation and HistoGel techniques. This can decrease the likelihood of adequate sample inclusion in the block. The automated Cellient system may also improve cell block quality compared with manual methods which depend to a greater degree on operator ability.

The reduced need for Cellient block recutting shown in this study has the potential to improve technologist and pathologist workflow and to reduce the time needed to render a diagnosis in clinical cases. In addition, the Cellient system has a rapid processing time of less than one hour compared with the 8-10 hours of processing required for the simple sedimentation and HistoGel methods. ${ }^{6}$ The use of CaSki cells, as in this study, is a limitation because cultured cell lines may not perform in the same way as clinical specimens. However, CaSki cells are well defined cancer cell lines that are extensively used to model clinical applications.

In conclusion, the Cellient system cell blocks allowed visualization of tracer cells at low concentrations that were not able to be detected using simple sedimentation or HistoGel cell blocks. The Cellient system cell block method offers the potential to improve detection of low cellularity specimens compared with traditional techniques.

\section{Acknowledgment}

The authors thank Laurie Cote and Norman Soule for their technical assistance in this research.

\section{Disclosure}

The authors are employees of Hologic Inc. Editorial support was provided by Jennifer E Layne.

\section{References}

1. Shivakumarswamy U, Arakeri SU, Karigowdar MH, Yelikar B. Diagnostic utility of the cell block method versus the conventional smear study in pleural fluid cytology. J Cytol. 2012;29(1):11-15.

2. Rowe LR, Marshall CJ, Bentz JS. Cell block preparation as an adjunctive diagnostic technique in ThinPrep monolayer preparations: a case report. Diagn Cytopathol. 2001;24(2):142-144.

3. Akalin A, Lu D, Woda B, Moss L, Fischer A. Rapid cell blocks improve accuracy of breast FNAs beyond that provided by conventional cell blocks regardless of immediate adequacy evaluation. Diagn Cytopathol. 2008;36(7):523-529.

4. Istvanic S, Fischer AH, Banner BF, Eaton DM, Larkin AC, Khan A. Cell blocks of breast FNAs frequently allow diagnosis of invasion or histological classification of proliferative changes. Diagn Cytopathol. 2007;35(5):263-269.

5. Sethi S, Geng L, Shidham VB, et al. Dual color multiplex TTF-1 + napsin A and p63 + CK5 immunostaining for subcategorizing of poorly differentiated pulmonary non-small carcinomas into adenocarcinoma and squamous cell carcinoma in fine needle aspiration specimens. Cytojournal. 2012;9:10.

6. Cellient automated cell block system operator's manual. Marlborough, MA: Hologic Inc; 2008.

7. ThinPrep 2000 operator's manual. Marlborough MA: Hologic Inc; 2007.

8. Thermo Scientific Richard-Allen Scientific HistoGel instructions for use. Kalamazoo, MI: Thermo Scientific; 2008.

9. Gorman BK, Kosarac O, Chakraborty S, Schwartz MR, Mody DR. Comparison of breast carcinoma prognostic/predictive biomarkers on cell blocks obtained by various methods: Cellient, formalin and thrombin. Acta Cytol. 2012;56(3):289-296.

10. Wagner DG, Russell DK, Benson JM, Schneider AE, Hoda RS, Bonfiglio TA. Cellient automated cell block versus traditional cell block preparation: a comparison of morphologic features and immunohistochemical staining. Diagn Cytopathol. 2011;39(10):730-736. 
Pathology and Laboratory Medicine International

\section{Publish your work in this journal}

Pathology and Laboratory Medicine International is a peer-reviewed, open access journal focusing on innovative basic research and translational research related to pathology or human disease. The journal includes original research, updates, case reports, reviews and commentaries on current controversies. The Academic Sponsor of this journal is the Chinese American Pathology Association (CAPA). The manuscript management system is completely online and includes a very quick and fair peer-review system. Visit http://www.dovepress.com/testimonials.php to read real quotes from published authors.

Submit your manuscript here: http://www.dovepress.com/pathology-and-laboratory-medicine-international-journal 